\title{
Sir Charles Sherrington y la naturaleza de lo mental
}

\section{Sir Charles Sherrington and the nature of mind}

\author{
CARLOS BLANCO \\ Universidad de Navarra
}

Recibido: 15/01/2013 Aprobado: 15/05/2013

\section{RESUMEN}

La figura del británico Sir Charles Scott Sherrington (1857-1952) ocupa un lugar privilegiado en la historia de la neurofisiología. Su principal contribución estriba en su descubrimiento de la «función integradora del sistema nervioso», en cuyo desarrollo se compendian sus importantes aportaciones al estudio de la diferenciación entre acciones inhibidoras y acciones excitadoras. Menos conocida resulta, sin embargo, su intensa pasión por la filosofía, por la historia (consagró una biografía al médico francés del siglo XVI Jean Fernel) y por la literatura (en especial, por la poesía de Goethe; él mismo compuso numerosos versos, publicados en obras como The Assaying of Brabantius and other Verse, de 1925). Sus amplias inquietudes filosóficas se plasmaron en el libro Man on His Nature, cristalización de las Gifford Lectures que impartió en la Universidad de Edimburgo entre mayo de 1937 y junio de 1938. Su profundidad conceptual y sus implicaciones para el debate contemporáneo en torno al problema mente-cerebro son insoslayables. En este trabajo nos detendremos, precisamente, en el análisis de la propuesta filosófica de Sherrington sobre la naturaleza de la mente humana.

PALABRAS CLAVE

SHERRINGTON, PROBLEMA MENTE-CEREBRO, CONCIENCIA, DUALISMO, PAMPSIQUISMO

\section{ABSTRACT}

Sir Charles Sherrington (1857-1952) has played a privileged role in the history of Neurophysiology. His main contribution is based on his discovery of the «integrative function of the 
nervous system», the development of which synthesizes some of his important experimental results concerning the study of inhibitory and excitatory actions. However, it is less known that Sherrington cultivated a deep passion for philosophy, history (he wrote a biography of the 16th century French physician Jean Fernel), and literature (especially, Goethe's poetry; Sherrington himself composed numerous verses, published in works like The Assaying of Brabantius and other Verse, de 1925). His broad philosophical interests were condensed in Man on His Nature, which contains his Gifford Lectures, given at the University of Edinburgh between May 1947 and June 1938. Its conceptual scope and its implications for the contemporary debate on the mind-body problem are analyzed in this paper, which aims to examine Sherrington's ideas about the nature of the human mind.

KEY WORDS
SHERRINGTON, MIND-BODY PROBLEM, CONSCIOUSNESS,
DUALISM, PAMSYCHISM

\section{SHERRINGTON, EL CIENTíFICO}

Los hallazgos Del neuroanatomista español Santiago Ramón y Cajal (1852-1934) sobre la estructura del sistema nervioso habían abierto un nuevo y brillante horizonte para el estudio científico del cerebro. Sir Charles Scott Sherrington ${ }^{1}$ protagonizaría la etapa «ulterior», caracterizada por el progresivo esclarecimiento de la fisiología de las células nerviosas. A él le debemos aportaciones pioneras a este campo, así como el término «sinapsis» (acuñado en 1897 a partir del griego sinapsis, «cerrar») para denominar el punto específico donde se comunican dos neuronas entre sí.

Sherrington, educado como médico en Londres, Edimburgo y Cambridge, se inclinó por el estudio de la fisiología después de leer las investigaciones de David Ferrier (1843-1928) sobre el córtex cerebral. En su etapa en Cambridge, Sherrington coincidió con Sir Michael Foster (1836-1907), autor de un influyente manual (A Textbook of Physiology; la primera edición data de 1877), y a la sazón director del departamento de fisiología de esta universidad. Foster había fundado la prestigiosa revista Journal of Physiology, en cuyas páginas se publicarían algunos de los artículos más importantes de la neurofisiología del siglo XX. Sherrington trabajó como ayudante de John Newport Langley (1852-1925), con quien editó algunos de sus primeros artículos. ${ }^{2}$ Posteriormente, nuestro hombre se trasladó como médico residente al «St. Thomas' Hospital» de Londres, para más tarde desplazarse hasta Bonn, donde estudió con Edouard Pflüger, renombrado experto en la acción refleja, y a Estrasburgo, donde co-

1 Para una biografía detallada de Sherrington, véase J.C. Eccles - W.C. Gibson, Sherrington: His Life and Thought.

2 J.N. Langley - Ch. S. Sherrington, «Secondary degeneration of nerve tracts following removal of the cortex of the cerebrum in the dog», 59-65. 
laboró con Friedrich Goltz (notoriamente opuesto a las tesis corticalistas de Ferrier). Sherrington dedicó algunos años al análisis del cólera a petición de dos sociedades británicas, ante la epidemia de cólera asiático que se había desatado en Europa meridional. Trabajó con Rudolf Virchow (1821-1902) y con Robert Koch (1843-1910), padre de la bacteriología médica y descubridor del bacilo de la tuberculosis, y en Berlín asistió a cursos de importantes neurofisiólogos, como Hermann von Helmholtz (1821-1894), Émile Du Bois-Reymond (18181896) y Wilhelm von Waldeyer (1836-1921).

En 1891, Sherrington fue nombrado profesor en la Brown Institution de la Universidad de Londres, donde continuó con sus investigaciones sobre patología e inmunología, si bien se centró, paulatinamente, en el sistema nervioso, rama a la que acabaría por consagrar todas sus energías. Uno de sus profesores en Cambridge, Walter Holbrook Gaskell (1847-1914), le aconsejó que se focalizara primero en el análisis de la médula espinal, a priori más simple y abordable que el examen del córtex cerebral. Ya René Descartes (1596-1650), en su tratado L'Homme, se había referido a la existencia de acciones reflejas, esto es, de movimientos involuntarios en los que no mediaba un acto consciente. El profesor de Oxford Thomas Willis (1621-1675), autor de la influyente obra Cerebri Anatome cui Accesit Nervorum Descriptio et Usus (1664), en la que sentaba las bases de la neuroanatomía moderna, asumió esta idea cartesiana. La explicó desde la hipótesis de que los espíritus animales (sutiles sustancias de naturaleza material que, tanto para Descartes como para la medicina clásica, recorrerían los nervios para transmitir las órdenes procedentes del cerebro a los órganos periféricos; las investigaciones de Luigi Galvani sobre la electricidad animal, publicadas a finales del siglo XVIII, demostrarían la inutilidad de la teoría de los espíritus animales) en el sistema nervioso central eran «reflejados» hacia los músculos inmediatamente después de tener lugar el estímulo. Sherrington estudió el reflejo en la rodilla con monos, conejos, gatos y perros, y apoyó la idea de Wilherlm Heinrich Erb (1840-1921), ${ }^{3}$ según la cual se trataba de un verdadero reflejo, asociado a la médula espinal. Logró, asimismo, identificar el mecanismo exacto de las neuronas motoras y sensoriales implicadas. ${ }^{4}$ Sherrington sugirió la existencia de órganos musculares especializados en el procesamiento de acciones reflejas. ${ }^{5}$ Muchos dudaban de la realidad de estas terminales de un «sexto sentido», pero lo cierto es que, con la formulación de

3 Cf. W. Erb, «Über Sehenreflexe bei Gesunden und Rückenmarkskranken», 792. Sobre las contribuciones de Erb a la neurociencia, cf. L. Sarikcioglu - R. Y. Arican, «Wilhelm Heinrich Erb (1840-1921) and his contributions to neuroscience», 732.

4 Ch. S. Sherrington, «Note on the knee-jerk», 145-147; «Note toward the localization of the knee-jerk», 545-654.

5 Ch. S. Sherrington, «The muscular sense», en E.A. Schäfer (ed.), Textbook of Physiology, vol. II, 1002-1025. 
su hipótesis, el científico británico había inaugurado un campo sumamente fecundo para la neurociencia del siglo XX: el estudio del sistema propioceptor.

En sus trabajos con monos, Sherrington destinó varios años al examen de los nervios espinales sensoriales y motores, para analizar las distribuciones anatómicas de las raíces espinales. ${ }^{6}$ En 1895 se trasladó al University College de Liverpool, y durante su período como docente e investigador en esa ciudad inglesa, Sherrington indagó, meticulosamente, en la inervación recíproca (planteada ya por Descartes en su De Homine, y explorada también por autores como Charles Bell -1774-1842-7 y Marshall Hall -1790-1857-8), así como en los mecanismos de coordinación entre los reflejos motores de inhibición y los de excitación. En 1896, tras descerebrar animales anestesiados, Sherrington fue capaz de desconectar el sistema nervioso inferior de los centros de organización cerebrales. Propició un estado de inconsciencia en los especímenes que le permitió estudiar las claves de la inervación recíproca. Comprobó, de esta manera, que al estimular una extremidad se producía coordinación motora, de tal forma que, automáticamente, se generaba movimiento en la extremidad opuesta, sin mediar una acción consciente. ${ }^{9}$

Los hallazgos de Sherrington sobre las acciones reflejas se condensaron, en gran medida, en su obra The Integrative Action of the Nervous System (1906),${ }^{10}$ fruto de las diez conferencias (las «Silliman Lectures») dictadas en 1904 en la Universidad de Yale. Sherrington comprendió que una característica fundamental del sistema nervioso reside en su función integradora (esto es, el propio sistema «integra» la información disponible y la «discrimina» de acuerdo con sus necesidades, para así emitir la respuesta adecuada). ${ }^{11} \mathrm{~A}$ juicio de la neurocientífica italiana Rita Levi-Montalcini (1909-...), ganadora del premio Nobel por su descubrimiento (junto con Stanley Cohen) del factor de crecimiento neuronal, la propiedad más importante del sistema nervioso estriba, precisamente, en este papel integrador, discernible ya en los celentéreos (las criaturas más antiguas dotadas de sistema nervioso). ${ }^{12}$ No menos

6 Ch. S. Sherrington, «Note on the arrangement of some motor fibres in the lumbosacral plexus», 621-772.

7 Ch. Bell, «On the nerves of the orbit», 289-703.

8 M. Hall, Synopsis of the Diastaltic Nervous System, de 1850.

9 Ch. S. Sherrington, «Decerebrate rigidity, and reflex coordination of movements», 319-332.

10 Sobre este importante trabajo de Sherrington, cf. J.P. Swazey, «Sherrington's concept of integrative action», 57-89; D.N. Levine, «Sherrington's The Integrative Action of the Nervous System: A centennial appraisal», 1-6.

11 Véase, sobre este concepto, E.R. Kandel - J.H. Schwartz - Th. M. Jessell, Principios de Neurociencia, 207-228.

12 R. Levi-Montalcini, La Galaxia Mente, 36. 
relevante, Sherrington se percató de que no todas las sinapsis son de naturaleza excitadora, sino que la mayoría exhibe cualidades inhibidoras, de tal manera que una neurona motora puede recibir impulsos excitadores o inhibidores. El adecuado entendimiento de la «inhibición» como proceso activo, y no simplemente como la ausencia de excitación, constituye una de las aportaciones más notables de Sherrington al estudio científico del sistema nervioso. Ramón y Cajal ni siquiera lo había intuido, y «no es fácil comprender por qué Cajal realizó todas sus interpretaciones funcionales sobre la base del flujo continuo de excitación, en que lo importante es 'la vía de conducción' (véanse las flechas en cualquiera de los dibujos de Cajal). Ningún indicio del concepto de inhibición. Una actividad que hoy sabemos representa una fracción mucho mayor que la de excitación, especialmente en aquellos centros cerebrales en los que tienen lugar procesos de integración»». ${ }^{13}$ Décadas más tarde, entre los años ' 50 y ' 60 , el neurofisiólogo australiano Sir John Eccles (1903-1997), alumno de Sherrington, elucidará los mecanismos iónicos mediante los cuales las neuronas motoras generan sus acciones inhibidoras y excitadoras (obtendrá, por ello, el premio Nobel de medicina o fisiología en 1963). ${ }^{14}$

Sherrington, en definitiva, advirtió que los animales poseedores de un sistema nervioso más desarrollado operan como un todo unificado en virtud de la integración central de la función nerviosa. Por sus contribuciones a la neurofisiología, Sherrington recibió el premio Nobel de medicina o fisiología en 1932, compartido con el también londinense Lord Edgar Adrian.

\section{SHERRINGTON, EL FILÓSOFO}

Era de esperar que un neurofisiólogo de la talla de Sherrington, quien albergó, en paralelo a su curiosidad científica, hondos intereses filosóficos, literarios e históricos, abordara en profundidad uno de los grandes interrogantes que aún hoy afronta la ciencia: la naturaleza de la mente humana. ${ }^{15}$ Ya en su Rede Lecture (establecidas en memoria de Sir Robert Rede, jurista del siglo XVI), titulada «The brain and its mechanism» y pronunciada en Cambridge el 5 de diciembre de 1933, ${ }^{16}$ Sherrington constata las dificultades asociadas a este problema, y muestra signos del planteamiento de tintes dualistas que caracterizará su propuesta filosófica. En palabras suyas, «las señales que entran

13 A. Ferrús, «La sinapsis, una unión necesaria entre Cajal y Sherrington», en A. Gamundí - A. Ferrús (eds.), Santiago Ramón y Cajal Cien Años Después, 176-177.

14 Véase J.C. Eccles, The Neurophysiological Basis of Mind. The Principles of Neurophysiology; y, del mismo autor, The Physiology of Synapses.

15 Para una síntesis de los escritos filosóficos de Sherrington, véase A. Zeman, «Sherrington's philosophical writings. A 'zest for life'», 1984-1987.

16 Ch. S. Sherrington, The Brain and Its Mechanism. 
en el cerebro no son mentales, como tampoco lo son las señales ejecutoras que generan. Pero la señalización que viaja a través de ciertas vías cerebrales [...] parece, por así decirlo, obtener energía mental, si bien la pierde de nuevo incluso antes de la penúltima vía de salida». ${ }^{17}$

En su exposición, Sherrington reconoce que sus frecuentes alusiones a nociones como «energía mental», «experiencia mental»y «eventos mentales» suscitarán no pocas suspicacias entre los científicos, pero manifiesta reticencia a abandonarlas; apego éste que perdurará en sus Gifford Lectures. Prefiere aventurarse a escrutar un posible mecanismo de correlación que mantenga la «realidad» de lo mental, sin subsumirlo en las estructuras y procesos neurofisiológicos subyacentes. La cuestión se refiere, como es lógico, al modo específico mediante el cual se lleva a cabo semejante «acoplamiento» entre lo mental y lo físico, porque, como él mismo admite en la citada conferencia, no existe indicio alguno de una diferencia fundamental entre las regiones no-mentales y las regiones mentales del cerebro en lo que concierne a las propiedades químico-físicas o a las estructuras microscópicas. Puede observarse cómo Sherrington insiste en la centralidad de lo «mental», hasta el punto de llegar a definir lo propiamente «físico» (o, como veremos más tarde, lo que incluirá bajo el denominado «principio energético») en oposición a lo mental. La «autonomía» de lo mental, o al menos su irreductibilidad, representa una constante en el pensamiento de nuestro autor. Este compromiso con la atribución de un cierto grado de sustancialidad a lo mental con respecto a lo físico goza de tal envergadura que, a su juicio, la incógnita sobre la interacción entre la mente y el cerebro no sólo carece de una respuesta convincente, sino de una «base» sólida, sobre cuyos pilares teóricos pueda siquiera presagiarse una solución futura. La dificultad más acuciante estribaría, por tanto, en la «inconmensurabilidad» entre lo físico y lo mental. La negativa de Sherrington a despachar lo mental como un proceso físico de elevada complejidad le obligará a explorar alternativas al fisicalismo, tal y como examinaremos más adelante.

Las ideas de Sherrington fueron rápidamente contestadas por otro ilustre científico: el ruso Ivan Pavlov (1849-1936), también ganador del premio Nobel de Medicina o fisiología (en 1904), y célebre por sus contribuciones al estudio del comportamiento animal (en particular de los reflejos condicionados). Pavlov, quien había recibido noticias de las palabras de Sherrington sobre la autonomía de lo mental pronunciadas en su Rede Lecture, criticó al británico en un seminario ofrecido en $1934 .{ }^{18} \mathrm{El}$ eje de sus reproches gravita en torno a

17 Palabras citadas por J.C. Eccles - W.C. Gibson, Sherrington. His Life and Thought, 106.

18 Sobre las divergencias entre Sherrington y Pavlov en torno a la naturaleza de la mente, véase L. Volicer, «Relationship between physiological research and philosophy in the work of Pavlov and Sherrington», 381-392; R. Granit, «Interactions between Pavlov and Sherrington», 182-186. 
la insinuación de Sherrington de que esclarecer la relación entre la actividad mental y la nerviosa quizás desborde los límites de la ciencia. A juicio de Pavlov, este comentario abre la puerta, peligrosamente, a una actitud dualista: «¿Cómo puede ser que a día de hoy un fisiólogo dude de la relación entre la actividad nerviosa y la mente?», se interroga, retóricamente, Pavlov. ${ }^{19}$ Para él, esta posición es subsidiaria de "un concepto puramente dualista», que concibe el cerebro como un instrumento pasivo y el alma como el «agente» que pilota el cuerpo. Es preciso advertir, sin embargo, que de las palabras de Sherrington no se colige necesariamente una óptica dualista de esta clase. El dualismo de Sherrington posee, como veremos a continuación, un mayor refinamiento teórico. Se asemeja más a un interaccionismo (como el que exhibirán, décadas más tarde, Sir John Eccles y Sir Karl Popper ${ }^{20}$ ). No conjetura la existencia

19 Palabras citadas por J.C. Eccles - W.C. Gibson, Sherrington. His Life and Thought, 106.

20 Sir John Eccles, además de sus importantes contribuciones a la ciencia (elucidó el mecanismo iónico de la inhibición postsináptica), desarrolló una profunda reflexión filosófica sobre el problema mente-cerebro, cristalizada, en gran medida, en el libro que escribió conjuntamente con el filósofo de la ciencia Sir Karl Popper, The Self and Its Brain. En él aboga por una perspectiva que recuerda bastante al dualismo interaccionista (la mente y el cuerpo como dos entidades independientes que interaccionan; cf. op. cit., 361-365) y, en ocasiones, al «trialismo» de Popper (la doctrina de los tres mundos: el mundo de los cuerpos físicos, el mundo mental o psicológico, y el mundo que comprende los productos de la vida humana; para un compendio de esta postura, véase K.R. Popper, Objective Knowledge). Sobre una síntesis de las ideas filosóficas de Sir John Eccles, remitimos a sus Gifford Lectures: The Human Mystery. Para una exposición de su propuesta de solución al problema mente-cerebro, consúltese J.C. Eccles, «A unitary hypothesis of mind-brain interaction in the cerebral cortex», 433-451, en la que formula una tesis netamente dualista (cuyas dificultades de cara a su aceptación científica son patentes): la interacción entre unidades neurales («dendrones», esto es, conjuntos de dendritas apicales de las células piramidales de las láminas V y III-II como unidades receptoras básicas) y unidades mentales (denominadas por Eccles «psicones»). Cada psicón (el cual contendría experiencias mentales características) se uniría de modo único a un dendrón. Esta tesis presenta dos problemas fundamentales: en primer lugar, resulta, por su propia naturaleza, incontrastable (con la misma legitimidad podríamos postular un psicón por cada dendrón que un único psicón que condensase todo el mundo mental); en segundo lugar, «hipostasia» lo mental como una sustancia en paralelo, para cuyo acceso sólo cabría un método introspectivo difícilmente verificable. Pese a ello, es de destacar que un neurofisiólogo de la altura de Sir John Eccles dedicase grandes esfuerzos a reflexionar (y a argumentar hipótesis más o menos discutibles, pero propuestas razonadas, al fin y al cabo) sobre una temática tan acuciante y de tan hondo calado en la historia del pensamiento filosófico y científico como la del problema mente-cerebro. Basten, en cualquier caso, las siguientes citas para poner de relieve la posición de Eccles en defensa de la autonomía «sustancial» del orden mental: «I maintain that the human mystery is incredibly demeaned by scientific reductionism, with its claim in promissory materialism to account eventually for all of the spiritual world in terms of patterns of neuronal activity. This belief must be classed as a superstition. . . . we have to recognize that we are spiritual beings with souls existing in a spiritual world as well as material beings with bodies and brains existing in a material world») 
de un «fantasma dentro de la máquina» (por apelar a la famosa metáfora de Gilbert Ryle), ${ }^{21}$ sino que postula la irreductibilidad última de lo mental, de manera que la actividad consciente dimanaría de la «sincronización» de lo mental y de lo físico.

Será en sus Gifford Lectures donde Sherrington profundice en el problema mente-cerebro y ofrezca su propio modelo. Las conferencias sobre teología natural auspiciadas por el jurista escocés Lord Gifford (1820-1887), quien otorgó una relevante donación económica a las universidades de Edimburgo, Glasgow, St. Andrews y Aberdeen para «promover y difundir el estudio de la teología natural en el sentido más amplio del término -en otras palabras, el conocimiento de Dios-»), han reunido a la flor y nata de la filosofía, de la teología y de la ciencia del siglo XX. De entre la nómina de conferenciantes, destacan filósofos como William James, Henri Bergson y Alfred North Whitehead, teólogos como Karl Barth, Albert Schweitzer y Jürgen Moltmann, y científicos como Niels Bohr, Werner Heisenberg y Sir John Eccles.

Como escriben Eccles y Gibson, cuando Sherrington impartió las Gifford Lectures, a finales de la década de los ' 30 , se albergaba confianza en la posibilidad de que «el hombre en todos sus caracteres mentales más señeros -pensamientos, imágenes, memorias, decisiones, creatividad en las artes y cienciasfuera explicable, en último término, de modo materialista y determinista». ${ }^{22} \mathrm{La}$ perspectiva de un monismo materialista, capaz de reducir la multiplicidad del fenómeno humano a su fundamento material, desterraría, por tanto, la unicidad de nuestra especie, su supuesto «privilegio» en el seno del cosmos físico (cuya verosimilitud había alimentado, en gran medida, el discurso religioso, y había nutrido una conspicua parte de la reflexión filosófica). Sherrington, consciente de la magnitud de las esperanzas depositadas en una comprensión puramente materialista de la mente humana, que contribuyera a «afianzar» la unidad de la visión científica del mundo (gracias a excluir la hipotética «excepcionalidad» humana), pronunció sus Gifford Lectures con la intención de plantear preguntas de enorme trascendencia, interrogantes que quizás pusieran en tela de juicio la plausibilidad de esa tentativa de «reducción» de lo mental a lo físico.

(Evolution of the Brain: Creation of the Self, 241); «since materialist solutions fail to account for our experienced uniqueness, I am constrained to attribute the uniqueness of the Self or Soul to a supernatural spiritual creation. To give the explanation in theological terms: each Soul is a new Divine creation which is implanted into the growing foetus at some time between conception and birth». (op. cit., 237); "we can regard the death of the body and brain as dissolution of our dualist existence. Hopefully, the liberated soul will find another future of even deeper meaning and more entrancing experiences, perhaps in some renewed embodied existence . . . in accord with traditional Christian teaching». (op. cit., 242).

21 G. Ryle, The Concept of Mind, 11-24.

22 J.C. Eccles - W.C. Gibson, Sherrington. His Life and Thought, 111. 
Sherrington, quien dedicará una documentada biografía al médico francés Jean François Fernel (1497-1558), uno de los fundadores de la fisiología moderna (autor de De Naturali Parte Medicinae, de 1542, y uno de los grandes sistematizadores de la medicina renacentista), ${ }^{23}$ utiliza la figura del humanista galo como uno de los hilos conductores que tejen la trama de sus ponencias. Para el científico británico, Fernel encarna un valioso ejemplo de una concepción armónica de la naturaleza y del espíritu, cuyos pilares quebrarán, de manera casi irreversible, con el advenimiento de la revolución científica y con la consolidación del espíritu de la modernidad. En ocasiones, las palabras de Sherrington traslucen nostalgia por ese equilibrio perdido, que parecía no abocar ni al monismo ni al dualismo, aunque nuestro autor reconoce que resultaría vano aspirar a recuperar, a día de hoy, una unidad reminiscente de la que predominó en la mente medieval y de la temprana modernidad, pues ésta no fue ajena a la superstición, así como a la ausencia de una indagación propiamente científica en la estructura y en el funcionamiento del universo material. Por otra parte, Sherrington cree que el progreso en la interpretación científica del mundo no tiene por qué apagar la llama de la sorpresa, del sobrecogimiento ante la belleza y la sofisticación que enardecen el universo, sino más bien avivarla. Cuanto más certeras y profundas se alzan las explicaciones que nos brinda la ciencia, más poder de fascinación se percibe en el engranaje de la naturaleza. ${ }^{24}$

Para Sherrington, el concepto de energía constituye la categoría central de la ciencia, su gran principio unificador, ${ }^{25}$ cuyo alcance permite explicarlo todo en términos físico-químicos (la biología se reduciría, así, a procesos físicoquímicos revestidos de mayor complejidad; no establecería, stricto sensu, un nuevo orden de lo real, en cuyo seno imperaran leyes sustancialmente distintas). Sin embargo, el «principio energético» no logra explicar la percepción subjetiva, el hecho de que yo perciba algo como «mío». Este momento de retorno, esta caracterización clásica de la conciencia como «vuelta sobre uno mismo», excede, a su juicio, los límites impuestos por la intelección científica del mundo, inspirada en el principio energético. En la contemplación del fulgor de una estrella, cuyo brillo colorea la inmensidad nocturna del firmamento, la ciencia nos proporcionará una explicación, en términos de patrones de energía, sobre cómo se produce su radiación, y sobre cómo, mediante una concatenación de procesos fotoquímicos, su luminiscencia genera en nuestro cerebro una imagen determinada, pero no revelará nuestra experiencia subjetiva en tanto que videntes de la estrella. La ciencia no puede penetrar en nuestra percepción, en 
lo que significa para cada uno de nosotros divisar la estrella en su centelleo. ${ }^{26}$ El esquema puramente energético que orienta la empresa científica aborda la estrella como un objeto de observación, no de percepción. No es apto para acceder a la experiencia mental, porque el acto mental no se palpa sensiblemente, sino que se experimenta subjetivamente. El yo no es un objeto. Su naturaleza subjetiva, privada, lo «oculta» para la observación empírica, pero no por ello deja de ser real. No consiste en una experiencia ilusoria, pues cada uno atesora vívidas memorias de su propio yo.

Para Sherrington, la mente no es susceptible de reducción a ninguna forma de energía (en la acepción «física» que subyace al concepto de «energía»; por tanto, toda apelación a una «energía mental» resultaría improcedente, si la noción de «energía» aquí empleada traspusiera la barajada por las ciencias naturales). La razón reside en la ausencia de espacio-temporalidad en los eventos mentales. Su inasibilidad, la incapacidad de experimentarlos en un tiempo y en un espacio (fenómeno que contrasta con su «realidad», con la efectividad de la conciencia, de las intenciones, de las voliciones...), implica que escapan, de un modo u otro, al dominio del principio energético. Se asemejan a entidades «infinitésimas», a límites asintóticos de los procesos materiales. Lo mental no obedece a una transformación de la energía, como en el caso de los eventos físicos. La ciencia no logra comprender lo mental desde los parámetros fijados por la noción de energía. Según Sherrington, en la cosmovisión científica, la mente parece proceder de la nada y retornar a la nada, ${ }^{27}$ en el sentido de que se antoja inasequible para una explicación puramente energética y evolucionista. Emerge misteriosamente desde lo no-mental, y cuando fallecen los individuos provistos de capacidades mentales, se sumerge en un enigmático y oscuro océano de nihilidad.

A la tentación platónica de concebir la mente como una entidad infinita aprisionada en un cuerpo finito, aherrojada en una estructura como la del cerebro, no sucumbe Sherrington, quien se cuida mucho de hablar de la «finitud» de la mente. Ésta recuerda a una especie de punto focal en movimiento, que vaga, restringidamente, dentro de cada uno de nosotros. ${ }^{28} \mathrm{La}$ experiencia sensible no es capaz de acceder a esta mente finita. A diferencia de lo que sucede con la vida, explicable en términos puramente físico-químicos, sin la necesidad de apelar (como en el vitalismo) a un «principio» o entelequia vital que establezca una frontera infranqueable entre la esfera abiótica y el orden biótico, en el caso de la mente existe un auténtico abismo: el que separa lo causal, lo

26 La cercanía de Sherrington con la posición que adoptará, décadas más tarde, Thomas Nagel es patente (véase Th. Nagel, «What is it like to be a bat?», 435-450).

27 Cf. Ch. S. Sherrington, Man on His Nature, 209.

28 Op. cit., 166-172. 
energético, lo determinado, de lo «intencional», de la clase de realidad en cuyo seno caben «fines». Es cierto que la «mente reconocible» (lo que normalmente entendemos por «mente»: sensaciones, cogniciones, voliciones...) surge siempre en conexión a actos motores, $\mathrm{y}$, según Sherrington, hubo de evolucionar en paralelo a una gradual integración motora en aquellos organismos dotados de un sistema nervioso más complejo, pero persiste una «irreductibilidad», un «límite», aparentemente asintótico, que preserva la autonomía de lo mental con respecto al ámbito de lo físico.

Tanto René Descartes como Thomas Willis y el mismo Sherrington realizaron aportaciones esenciales a la reflexología, esto es, al estudio de las acciones involuntarias, pero es interesante percatarse de que estos tres grandes científicos se afanaron, tenazmente, en preservar la irreductibilidad de lo mental. En ningún momento propusieron explicar la actividad mental, ni la versatilidad del comportamiento humano, como resultados de procesos reflejos, en último término inconscientes, sino que les atribuyeron una autonomía sobre lo físico. La distinción entre lo mental y lo material es, para Sherrington, incuestionable. Con todo, nuestro autor sostiene que la experiencia mental, «aun totalmente dispar de todos los eventos materiales y por tanto del acto físico», no se desliga por completo de lo que él engloba dentro de la categoría de «principio energético»: lo mental y lo energético proceden, «de alguna manera», conjuntamente. ${ }^{29}$

Sin embargo, se requiere de ulteriores matizaciones, porque ¿adopta aquí Sherrington un paralelismo psicofísico à la Leibniz, tesis que postula una misteriosa sincronización de lo material y de lo mental decretada por una autoridad ignota? ¿Se adhiere a una posición determinista como la de Thomas Huxley (1825-1895), para quien lo físico condiciona inexorablemente lo mental, si bien lo mental goza de «autonomía entitativa»? ${ }^{30}$ ¿Se refiere a un dualismo interaccionista clásico, como el de Descartes, o a uno más sofisticado, como el que propondrán Sir John Eccles y Sir Karl Popper? La posición de Sherrington no se esclarece con facilidad. La mente acompaña la acción motora, pero lo que le incumbe no es el acto, sino el propósito. ${ }^{31}$ La mente, en definitiva, no corresponde a la materialización de la acción. El problema reside en el dualismo subyacente a este planteamiento. Si la mente se encarga de los «fines», de la teleología de la acción, ello se debe a que existe un profundo hiato entre lo puramente físico, regido por el principio de causalidad, y un enigmático universo mental en el que son posibles las «intenciones», los

29 Op. cit., 149.

30 Cf. Th. Huxley, «Bishop Berkeley on the metaphysics of sensation», en Th. Huxley (ed.), Hume with Helps to the Study of Berkeley, 251-252.

31 Ch. S. Sherrington, Man on His Nature, 170. 
«propósitos», la «indeterminación». El concepto de intencionalidad de Franz Brentano (1838-1917) ${ }^{32}$ y de numerosos autores de la escuela fenomenológica formula, con gran concisión, aquello a lo que alude Sherrington al invocar la categoría de «propósito».

Para Sherrington, la mente no pudo irrumpir de novo en la dinámica evolutiva de la vida: «la mente humana constituye un producto reciente por parte de nuestro planeta, generado por una mente que se encontraba allí desde mucho antes, y que suscitó la mente humana a través de transformaciones graduales de la mente previa». ${ }^{33}$ En el párrafo anterior resulta inevitable contemplar la rúbrica de una posición pampsiquista: la mente «ha existido desde el inicio», al menos en paralelo a la materia, pero en virtud de un mecanismo sumamente arcano ha convergido, en todo momento, con la evolución de la materia hacia cotas de mayor complejidad; desarrollo éste que se ha traducido en la adquisición de un sistema nervioso más integrado. Se trata de una propuesta bastante similar a la de pensadores como el matemático y filósofo británico William Clifford (1845-1879) 34 y el paleontólogo y teólogo francés Pierre Teilhard de Chardin (1881-1955). ${ }^{35}$ Las dificultades saltan a la vista: ¿cómo aconteció semejante convergencia? ¿Cómo es posible que lo mental haya evolucionado en paralelo a lo físico? ¿Por qué sólo se manifiesta cuando lo físico corona unos niveles de complejidad tan notables como los conquistados por la evolución de los mamíferos superiores? ¿Es legítimo aseverar que existe un psiquismo subyacente a todas las formas de vida, e incluso a las estructuras abióticas, que permanecería «latente» hasta la emergencia de los seres más evolucionados?

Pese a éstas y a otras objeciones pertinentes a la propuesta de Sherrington, es preciso reconocer que el gran neurofisiólogo británico asume un pampsiquismo coherente con la teoría de la evolución. A diferencia de una idea «estática» de la mente, como la que parece inferirse de la obra cartesiana, Sherrington no tiene reparos en admitir una evolución de lo mental. Y, en efecto, si la visión científica del mundo natural posee, como uno de sus

32 Una obra clave para la comprensión de la noción de intencionalidad en el pensamiento de Brentano es su Psychologie vom empirischen Standpunkt. Para Brentano, todo fenómeno mental viene caracterizado por una «intencionalidad», esto es, por una referencia a un contenido en forma de «objetividad inmanente». Existe, por tanto, una nítida distinción entre los fenómenos físicos y lo de índole mental. La idea de intencionalidad, de indudable valor en el estudio de la mente, suscita, sin embargo, no pocos interrogantes, porque establece una barrera, prácticamente infranqueable, entre $m i$ experiencia del mundo, el mundo, y las experiencias ajenas del mundo. En cualquier caso, el concepto de intencionalidad remite al núcleo mismo del problema mente-cerebro.

33 Ch. S. Sherrington, Man on His Nature, 173.

34 Véase W. K. Clifford, «On the Nature of Things-in-Themselves», 57-67.

35 Consúltese, en especial, su obra El Fenómeno Humano. 
ejes vertebradores, el concepto de «evolución» de la vida (la evolución de la materia hacia formas más complejas, según los requisitos de adaptación que experimentan determinadas especies), el gran neurofisiólogo británico aplica esta noción también al esquivo universo de la mente. La evolución de lo mental sería tan incontestable como la de lo corpóreo. La mente humana se hallaría, de hecho, sumida en un estado de innegable incompletitud, sin que sepamos si en algún momento alcanzará algo así como una «consumación». ${ }^{36}$

La inconmensurabilidad entre lo mental y lo físico se expresa en su divergente modo de operar. Mientras que lo físico obedece al «principio energético», esto es, a patrones de intercambio de energía (todo trabajo que se efectúe en el seno del mundo material exige energía: ésta es, precisamente, una de las dificultades más poderosas que encara toda perspectiva dualista), lo mental responde a una dinámica totalmente distinta. Los patrones de energía acaecen en el espacio y en el tiempo. Son dimensionales; por tanto, se «localizan» en un punto y en un instante. La mente no exhibe esta naturaleza dimensional. No se «encuentra» en ubicación alguna ni se palpa en un momento concreto. Los acontecimientos físicos y los eventos mentales no son, por tanto, reductibles los unos a los otros, porque el «yo» jamás se aprehende en una localización espacio-temporal específica. Este hecho, que a no pocos autores induciría a desdeñar la existencia de algo así como un «yo», para considerarlo una ficción, ${ }^{37}$ brinda, para Sherrington, un argumento a favor de la autonomía de lo mental. La mente no puede proceder de la vasta evolución de la materia. No puede brotar de la no-mente. La evolución construye entidades provistas de mayor complejidad basada en lo que ya existe, pero la mente pertenece a una categoría «sui generis». No se asemeja a la energía física. Aunque haya evolucionado desde niveles revestidos de una menor complejidad, este proceso evolutivo parte ya de la mente, no de la materia. Hubo de subsistir una mente más elemental con anterioridad a que brotara una más compleja. La evolución

36 Cf. Ch. S. Sherrington, Man on His Nature, 260-294.

37 Ésta parece ser la posición de Daniel Dennett en La Conciencia Explicada: Una Teoría Interdisciplinar, 289, quien cita unas conocidas palabras de David Hume, procedentes de su Tratado sobre la Naturaleza Humana: «En lo que a mí respecta, siempre que penetro más íntimamente en lo que llamo mí mismo tropiezo en todo momento con una u otra percepción particular; sea de calor o frío, de luz o sombra, de amor u odio, de dolor o placer. Nunca puedo atraparte a mí mismo en ningún caso sin una percepción, y nunca puedo observar otra cosa que la percepción (...). Si tras una reflexión seria y libre de prejuicios hay alguien que piense que él tiene una noción diferente de sí mismo, tengo que confesar que yo no puedo seguirle en sus razonamientos. Todo lo que puedo concederle es que él puede estar tan en su derecho como yo, y que ambos somos esencialmente diferentes en este particular. Es posible que él pueda percibir algo simple y continuo a lo que llama su yo, pero yo sé con certeza que en mí no existe tal principio». 
cerebral, para Sherrington, «toma» la mente elemental y la desarrolla hasta niveles de complejidad creciente.

Los observadores críticos quizás aduzcan que Sherrington arriba a estas conclusiones como consecuencia de haber adoptado una distinción demasiado rígida y abrupta entre lo mental y lo no-mental. Lo mental bien pudiera provenir de la paulatina evolución de la materia hacia manifestaciones más complejas. Sherrington replicaría que esta posición se ve obligada a negar la especificidad de lo mental, constreñido ahora a una especie de epifenómeno que se desencadena en los niveles más complejos de la organización material. En coherencia, lo mental debería «ubicarse» espacio-temporalmente, y tratarse desde la perspectiva del principio energético, de los intercambios de energía como motores que subyacen a la totalidad de los procesos del mundo material; postura ésta, a su juicio, inadecuada para dar cuenta de la riqueza de los eventos propiamente mentales. No basta con acumular, aditivamente, células al cerebro para explicar la actividad mental: «la organización celular del cerebro puede representar la clave del secreto de su correlación con la mente, pero no parece que lo haga por la dotación mental individual de cada una de sus células constitutivas». Se aprecia aquí la proximidad entre las ideas de Sherrington y las de su eminente compatriota Alfred Russell Wallace (1823-1913), co-descubridor, junto con Charles Darwin, de la evolución por selección natural: «si un elemento material, o la combinación de mil elementos materiales en una molécula, son igualmente inconscientes, nos es imposible creer que la mera adición de uno, dos u otros mil elementos materiales para formar una molécula más compleja pudiera, en modo alguno, llevar a producir una existencia autoconsciente. No hay manera de escapar al dilema: o toda la materia es consciente, o la conciencia es o pertenece a algo distinto de la materia». ${ }^{38}$ De nuevo, para autores como Wallace y Sherrington, esta frontera tan radical que escinde la «realidad física» de la mental carece de analogía con la divisoria que separa lo vital de lo inerte. Sólo la mente, por su naturaleza «irreductible» a procesos de transformación energética, escapa al dominio de la visión científica del mundo.

Sin embargo, Sherrington no logra desentrañar un interrogante que persiste, a nuestro juicio, indómito: ¿cómo se unifica exactamente esa multiplicidad de procesos neuronales que concurren en una experiencia perceptiva singular?

38 A.R. Wallace, Natural Selection and Tropical Nature, 209. Es interesante observar que, de acuerdo con el argumento de Wallace, tampoco cabría explicar, científicamente, la capacidad de auto-movimiento que poseen los seres «animados»: si un átomo o una molécula carecen de esta propiedad, cuesta creer que, por la simple adición de más elementos materiales, emerjan características como la motilidad o la sensibilidad. Sin embargo, la ciencia ha logrado dar razón de éstas y de otras muchas propiedades desde su fundamento físico-químico. Subsiste, es cierto, el profundo misterio de la conciencia, pero escudarse en la ausencia de esta facultad en los elementos materiales no basta para atribuirle un carácter entitativo «de suyo». 
Ese hipotético yo, ¿cómo ejerce su acción integradora sobre el cerebro? ¿La aplica a una estructura cortical en particular, u opera simultáneamente sobre diversas regiones? Y, de nuevo, si el yo no se localiza espacialmente, pero estriba en una convergencia temporal (como una especie de «sentido interno» kantiano), ¿por qué atribuirle inmaterialidad, si lo temporal posee carácter material, «dimensionalizado»?

Por otra parte, ¿cabe hablar de una postura funcionalista en Sherrington? Parece que si por funcionalismo ${ }^{39}$ nos referimos a la «hipostatización» de los estados mentales como funciones de una estructura cerebral, al modo de un software instalado en un hardware, la tesis de Sherrington dista entonces considerablemente de este planteamiento. El funcionalismo, al menos en sus versiones más difundidas, no implica (quizás sí en la práctica, pero desde luego no en la teoría) una cosmovisión dualista, que remita a la inconmensurabilidad entre el orden de lo mental y la esfera de lo físico. La mente, en el funcionalismo, no constituye una entidad sui generis e inmaterial, mientras que los eventos mentales, para Sherrington, difieren cualitativamente de los procesos regidos por intercambios de energía (esto es, del dinamismo de la naturaleza, en el sentido más laxo). Lo mental no puede explicarse como una forma de materiaenergía. Esta convicción es tan profunda en Sherrington que nuestro autor llega a sostener que la fisiología cerebral poco tiene que ofrecerle a la psiquiatría, pues «lo mental no se puede examinar como una forma de energía. Ésta es, en resumen, la brecha que divide la psiquiatría de la fisiología». ${ }^{40}$ Sorprende, en cualquier caso, que en sus Gifford Lectures Sherrington acuse a Sigmund Freud de no haber prestado, en la elaboración de sus teorías psicoanalíticas, la suficiente atención a la anatomía, ${ }^{41}$ cuando él mismo admite que esta disciplina poco coadyuvará al estudio de la mente, ya que subsiste un hiato invencible entre lo mental y lo material.

Sherrington apuesta por un nítido dualismo mente-cerebro, ${ }^{42}$ que postula una evolución paralela, y perfectamente sincronizada, de lo mental con lo corporal. La categoría de «simultaneidad» adquiere gran importancia en su modelo, no sólo en lo que respecta al «acoplamiento» de ambos procesos evolutivos, sino a la explicación de la concurrencia de mente y cerebro en toda actividad en cuyo decurso ambos sean partícipes. La convergencia de lo mental y de lo cerebral no posee naturaleza espacial, sino temporal. La mente «no se halla en ningún sitio». Resulta inasequible a toda tentativa de localiza-

39 Sobre el funcionalismo en relación al problema mente-cerebro, véase H. Putnam, «The nature of mental states», en W.H. Capitan - D.D. Merrill (eds.), Art, Mind and Religion, 37-48.

40 Ch. S. Sherrington, Man on His Nature, 228.

41 Op. cit., 189.

42 Cf. J.C. Eccles - W.C. Gibson, Sherrington: His Life and Thought, 135. 
ción espacial, como Sherrington ya ha apuntado. Su correlación con el cerebro consiste en una «síntesis temporal». ${ }^{43}$ En este punto, su cercanía a la filosofía de Kant es patente: el yo se concibe como una síntesis, como una unidad en el tiempo. No es susceptible de disgregarse en percepciones particulares (para Kant, el ich denke unifica la multiplicidad de sensaciones desde unos patrones de inteligibilidad que él mismo «pone»). ${ }^{44}$ El sujeto constituye una unidad irreductible, que no se manifiesta espacial sino temporalmente, en virtud de su actividad «sintética». ${ }^{45}$ Para que acaezcan eventos mentales, lo que se necesita es una confluencia temporal entre la mente y los procesos neurofisiológicos que condicionan este dinamismo. Tal y como escribe Sir John Eccles, la tesis de Sherrington sugiere que «existe un nivel de independencia de las experiencias mentales con respecto a los eventos cerebrales». ${ }^{46}$

El problema de la anterior tesis salta a la vista: ¿cómo, exactamente, unifica el yo la multiplicidad de sus sensaciones en una percepción consciente, subjetiva? ¿Cómo, en definitiva, se produce la interacción entre un hipotético yo, ajeno a la categoría de espacio, y los procesos cerebrales? ¿Qué gana la ciencia al postular la existencia de una hipóstasis subjetiva que actúe en paralelo a los circuitos cerebrales, si esta enigmática entidad consiste, en el fondo, en una síntesis temporal, explicable materialmente como la convergencia, en un instante dado, de distintos procesos neurofisiológicos que generan percepciones subjetivas? El yo de Sherrington «se impone», de alguna manera, sobre las redes neuronales que operan en el cerebro. Actúa como una cúspide integradora que ejerce su causalidad «de arriba abajo». La razón que mueve a Sherrington a adoptar esta aproximación ya la hemos mencionado: la irreductibilidad de la percepción subjetiva a procesos puramente físicos.

\section{CONCLUSIONES}

La postura de Sherrington sobre la relación entre la mente y el cerebro es palmariamente dualista, cercana a la que abrazarán discípulos suyos como Wilder Penfield ${ }^{47}$ (1891-1976) y Sir John Eccles. Impregnada de una perspectiva

43 Ch. S. Sherrington, Man on His Nature, 216ss.

44 La perspectiva de Kant se encuentra desarrollada en su Crítica de la Razón Pura.

45 Se trata, por otra parte, de una tesis que también encuentra eco en las filosofías de inspiración fenomenológica. Así, Maurice Merleau-Ponty escribe: «Je ne suis pas le résultat ou l'entrecroisement des multiples causalités qui déterminent mon corps ou mon 'psychisme'(...). Tout ce que je sais du monde, même par science, je le sais à partir d'une vue mienne ou d'une expérience du monde sans laquelle les symboles de la science ne voudraient rien dire rien» (Phénoménologie de la Perception, en CEuvres, 658).

46 J.C. Eccles - W.C. Gibson, Sherrington: His Life and Thought, 129.

47 Cf. W. Penfield, The Mystery of the Mind: A Critical Study of Consciousness and the Human Brain. En palabras de Penfield, sus investigaciones, y en especial su trabajo con pacientes 
pampsiquista, atribuye a lo mental una cierta «preexistencia», que surcaría toda la historia cósmica y evolucionaría en paralelo al desarrollo de las formas materiales. Para el gran científico británico, la conjunción de mecanismos cerebrales no es capaz de generar un evento mental. Ha de postularse la existencia de lo mental en paralelo a la de lo físico. Lo mental no se concentra en una única región del cerebro, o en una «célula pontificia» que controle, en último término, el proceso, como clímax de la integración nerviosa. No existe una unidad superior de integración en el terreno material que «conecte» directamente con lo mental, reminiscente de la glándula pineal cartesiana, sino que lo mental se diluye en una enorme expansión en millones de células.

El mundo, para Sherrington, se resuelve en dos clases de realidad: energética y mental; una energía espacio-temporal sensible y una mente insensible e inextensa (puramente «intensiva»). Entre ambas, nuestro autor aprecia una íntima correlación, pero no parece existir, a nivel estructural, una diferencia notable entre una región cerebral estrechamente ligada a la actividad de la mente y otra no tan nítidamente vinculada a ella. En su materialidad, las distintas regiones del cerebro se construyen a partir de los mismos elementos y operan guiadas por leyes físico-químicas comunes. No cabe, por tanto, identificar lo mental (experimentado, vivamente, en las facultades superiores del psiquismo humano) con tal o cual área del cerebro. Esta consideración de Sherrington cobraría especial importancia en nuestros días, cuando las técnicas de neuroimagen, cuyo desarrollo ha desvelado tanta información sobre la especialización funcional de las diversas regiones del cerebro, han adquirido un rol prominente en los debates sobre la naturaleza de la conciencia. Para Sherrington, elucidar la funcionalidad de una región cerebral determinada no eclipsa la evidencia de la inconmensurabilidad entre lo mental y lo físico, así como la constatación de que, a nivel puramente estructural, las diversas áreas no difieren sustancialmente entre sí. Lo mental ha de responder, por tanto, a un «principio» distinto, sui generis, irreductible a la concurrencia de procesos materiales. Lo material y lo mental constituyen dos órdenes dispares, no convertibles el uno en el otro. Utilizan lenguajes distintos: el de la energía, por un lado, y el de la conciencia (o «percepción»), por otro.

Sin embargo, la hipótesis de Sherrington genera, en nuestra opinión, objeciones demasiado serias. Tal y como hemos mostrado, la principal dificultad se refiere a la falta de clarificación sobre cómo, exactamente, interaccionan (o, en terminología sherringtoniana, «se correlacionan») lo mental y lo físicoenergético. Por otra parte, Sherrington parece concebir lo mental como una síntesis temporal, como una «intensión» ajena al espacio, que se manifiesta

epilépticos, lo condujeron a adoptar la hipótesis de que «nuestro ser consta de dos elementos fundamentales [mente y materia]». (Op. cit., 80). 
en la sincronización de la actividad cerebral con un hipotético yo, irreductible a los procesos neurofisiológicos. Con todo, una problemática análoga a la que plantea la independencia («no-localización») espacial del yo viene suscitada por su dependencia temporal. El yo de Sherrington, al igual que permanece inasequible a una caracterización espacial, debería ser «atemporal», porque el tiempo representa una dimensión de la materia y es constitutivo, como el espacio, de lo que nuestro autor ha denominado «principio energético», ley fundamental de la naturaleza. Si ese yo consiste simplemente en una sincronización temporal de procesos cerebrales, ¿por qué ha de apelarse a una hipóstasis mental? Si se desea sugerir que dicha hipóstasis mental cristaliza en la sincronización de determinados procesos cerebrales, ¿no resulta tanto o más confuso entender cómo puede plasmarse una entidad inmaterial en un instante temporal?

Esta aporía la encontramos también en la noción sherringtoniana de «evolución de la mente», sin duda un intento honesto de dotar la tesis dualista de verosimilitud ante la moderna teoría de la evolución de las especies. Si lo mental es inmaterial, eximido de la obediencia al «principio energético», no se comprende fácilmente cómo es factible que evolucione, pues todo proceso evolutivo conlleva transformaciones espacio-temporales en términos de intercambios energéticos. ¿A qué se refiere entonces Sherrington al invocar una evolución de la mente? Si lo mental es irreductible a lo material, y radica en una especie de «límite asintótico», infinitésimo, cuyo fundamento trasciende lo espacio-temporal y unifica la multiplicidad de la sensación en una unidad perceptiva, comportará siempre un «punto», inconmensurable a la dinámica material, y, por tanto, ajeno al «cambio». La supuesta evolución mental implicaría una creciente convergencia entre el cerebro y esa unidad mental irreductible, pero sólo se modificaría la entidad cerebral, cuya adquisición de formas más complejas permitiría una mejor sincronización (o correlación) con ese yo inasible, inextenso e «insensible». Sherrington, de ser coherente con su posición, debería aludir no tanto a una evolución pampspiquista de lo mental como a una confluencia gradual entre la materia y esa unidad mental «inmutable» (dado que es inmaterial), gracias a la progresiva evolución de las estructuras corporales hacia niveles de mayor complejidad y de una más refinada «concentración» funcional.

El dualismo de Sherrington establece una profunda fisura en el seno de la realidad. Su fragmentación irreconciliable entre la materia y la mente dificulta en extremo (o más bien imposibilita) vislumbrar una explicación científica de la conciencia. Lo cierto es que el hecho de que un científico de la talla de Sherrington, una de las cimas de la fisiología del siglo XX, se haya adherido a un planteamiento dualista muestra, de manera suficientemente expresiva, la gravedad del problema que afronta la ciencia a la hora de elucidar la naturaleza de lo mental. Aunque el argumento de autoridad quizás sea el menos autoritativo 
de los argumentos, resulta innegable que, cuando neurocientíficos de la altura de Sherrington, Penfield y Eccles adoptan un posicionamiento dualista, su actitud se debe a la persistencia de un misterio hondo y aparentemente inabordable: cómo puedo yo "percatarme de mí mismo», es decir, poseer conciencia. Los trabajos realizados en las últimas décadas han desvelado, gracias a avances técnicos como, por ejemplo, la neuroimagen, la funcionalidad de las distintas áreas cerebrales. El estudio de la relación entre conciencia y lenguaje ha puesto de relieve la estrecha conexión que existe entre ambos. La fisiología, por su parte, ha avanzado considerablemente en el estudio de distintos sistemas sensoriales (aunque subsista el denominado «binding problem»). Sin embargo, descifrar las claves de la conciencia constituye, aún hoy, el mayor desafío para la visión científica del mundo.

\section{REFERENCIAS BIBLIOGRÁFICAS}

BELL, Ch. «On the nerves of the orbit», Philosophical Transactions of the Royal Society 113 (1823), 289-703.

BRENTANO, F. Psychologie vom empirischen Standpunkt (Duncker \& Humblot, Leipzig), 1874.

CLIFFORD, W.K. «On the Nature of Things-in-Themselves», Mind 9/3 (1878), 57-67.

DENNETT, D. La Conciencia Explicada. Una Teoría Interdisciplinar, Paidós, Barcelona 1995.

ECCLES, J.C. The Neurophysiological Basis of Mind. The Principles of Neurophysiology, Clarendon Press, Oxford 1953.

—, The Physiology of Synapses, Academic Press, Nueva York 1964.

—, Popper, K.R. The Self and Its Brain, Springer International, Berlín 1977.

_, Gifford Lectures: The Human Mystery, Springer, Berlín 1979.

—, Evolution of the Brain: Creation of the Self, Routledge, Londres 1989.

-, «A unitary hypothesis of mind-brain interaction in the cerebral cortex», Proceedings of the Royal Society of London B 240 (1990), 433-451.

—, Popper, K.R. The Self and Its Brain, Springer International, Nueva York 1977.

GIBSON, W.C. Sherrington. His Life and Thought, Springer International, Berlín 1979.

ERB, W. «Über Sehenreflexe bei Gesunden und Rückenmarkskranken», Archiv für Psychiatrie und Nervenkrankheit 5 (1875), 792.

FERRÚS, A. «La sinapsis, una unión necesaria entre Cajal y Sherrington», en A. Gamundí - A. Ferrús (eds.), Santiago Ramón y Cajal Cien Años Después, 176-177.

GRANIT, R. «Interactions between Pavlov and Sherrington», Trends in Neuroscience 5 (1982), 182-186.

HALL, M. Synopsis of the Diastaltic Nervous System, J. Mallett, Londres 1850.

HUXLEY, Th. «Bishop Berkeley on the metaphysics of sensation», en Th. Huxley (ed.), Hume with Helps to the Study of Berkeley, Macmillan, Londres 1897, 251-252.

KANDEL, E.R. - SCHWARTZ, J.H. - JESSELL, Th. M. Principios de Neurociencia, McGraw Hill, Madrid 2001. 
KANT, I. Crítica de la Razón Pura, Alfagurara, Madrid 2000.

LANGLEY, J.N. - SHERRINGTON, Ch. S. «Secondary degeneration of nerve tracts following removal of the cortex of the cerebrum in the dog», Journal of Physiology, 5 (1884), 59-65.

LEVI-MONTALCINI, R. La Galaxia Mente, Crítica, Barcelona 2000.

LEVINE, D.N. «Sherrington's The Integrative Action of the Nervous System: A centennial appraisal», Journal of the Neurological Sciences 253 (2007), 1-6.

MERLEAU-PONTY, M. Phénoménologie de la Perception, en CEuvres, Gallimard, París 2010.

NAGEL, Th. «What is it like to be a bat?», Philosophical Review 83/4 (1974), 435-450.

PENFIELD, W. The Mystery of the Mind: A Critical Study of Consciousness and the Human Brain, Princeton University Press, Princeton NJ 1975.

POPPER, K.R. Objective Knowledge, Clarendon Press, Oxford 1972.

PUTNAM, H. «The nature of mental states», en W.H. Capitan - D.D. Merrill (eds.), Art, Mind and Religion, Pittsburg University Press, Pittsburg 1967.

RYLE, G. The Concept of Mind, Barnes \& Noble, Nueva York 1949.

SARIKCIOGLU, L. - Arican, R.Y. "Wilhelm Heinrich Erb (1840-1921) and his contributions to neuroscience», Journal of Neurology, Neurosurgery and Psychiatry 78/7 (2007), 732.

SHERRINGTON, Ch. S. «Note on the knee-jerk», St. Thomas' Hospital Reports 21 (1891), 145-147.

—, «Note toward the localization of the knee-jerk», British Medical Journal 1 (1892), 545-654.

-, «Note on the arrangement of some motor fibres in the lumbosacral plexus», Journal of Physiology 13 (1892), 621-772.

—, «Decerebrate rigidity, and reflex coordination of movements», Journal of Physiology 12 (1898), 319-332.

—, «The muscular sense», en E.A. Schäfer (ed.), Textbook of Physiology, Young J. Pentland, Edimburgo 1900, vol. II, 1002-1025.

-, The Brain and Its Mechanism, Cambridge University Press, Cambridge 1933.

—, Man on His Nature, Cambridge University Press, Cambridge 1940.

—, The Endeavour of Jean Fernel, Cambridge University Press, Cambridge 1946.

SWAZEY, J.P. «Sherrington's concept of integrative action», Journal of the History of Biology 1 (1968), 57-89.

TEILHARD DE CHARDIN, P. El Fenómeno Humano, Taurus, Madrid 1967.

VOLICER, L. «Relationship between physiological research and philosophy in the work of Pavlov and Sherrington», Perspectives in Biology and Medicine 16/3 (1973), 381-392.

WALLACE, A.R. Natural Selection and Tropical Nature, Macmillan, Londres 1870.

ZEMAN, A. "Sherrington's philosophical writings. A 'zest for life'», Brain 130/8 (2007), 1984-1987. 
Carlos Blanco es investigador y profesor en el Instituto de Cultura y Sociedad de la Universidad de Navarra.

Linea de investigación

Teoría del conocimiento, historia de las ideas y filosofía de la ciencia.

Publicaciones recientes

Conciencia y Mismidad (Dykinson, 2013).

Vida, interioridad y lucha: para una definición de la vida en diálogo con Helmut Plessner y Hans Jonas (Ideas y Valores (2013), 129-141)

Historia de la Neurociencia (Biblioteca Nueva, en prensa).

Dirección electrónica: carlosblanco@unav.es 
